\title{
e-Migrinter
}

17 | 2018

Enseigner les migrations internationales

\section{Critique du transnationalisme}

Un regard sur la formation en sociologie des migrations à l'URMIS

\section{Swanie Potot}

\section{(2) OpenEdition}

\section{Journals}

Édition électronique

URL : https://journals.openedition.org/e-migrinter/1054

DOI : 10.4000/e-migrinter.1054

ISSN : 1961-9685

Éditeur

UMR 7301 - Migrinter

Référence électronique

Swanie Potot, « Critique du transnationalisme », e-Migrinter [En ligne], 17 | 2018, mis en ligne le , consulté le 20 mai 2021. URL : http://journals.openedition.org/e-migrinter/1054 ; DOI : https://doi.org/ 10.4000/e-migrinter.1054

Ce document a été généré automatiquement le 20 mai 2021.

Tous droits réservés 


\title{
Critique du transnationalisme
}

\author{
Un regard sur la formation en sociologie des migrations à l'URMIS
}

\section{Swanie Potot}

1 Ce nouveau tournant dans la sociologie des migrations offre l'occasion d'un retour critique sur l'évolution des théories qui ont façonné ce champ de recherche. Celle-ci fut saisie lors du colloque célébrant les 30 ans du laboratoire Migrinter $^{1}$, au cours duquel une session portait sur l'enseignement de l'étude des migrations en France. L'unité de recherche migrations et société (URMIS, UMR 8245) est, avec le laboratoire cité, l'autre centre de recherche français très tôt spécialisé dans le domaine des migrations ${ }^{2}$. En ce sens, les formations de troisième cycle qui y sont dispensées depuis plus de vingt ans, à Paris et à Nice, sont révélateurs des évolutions de ce champ. Un regard rétrospectif sur les théories enseignées dans les masters de cette unité permet non seulement de retracer l'évolution d'un champ mais également d'ouvrir la porte à un débat épistémologique avec lequel les étudiants sont amenés à se familiariser au cours de leur parcours. On considère en effet que la formation à la recherche ne consiste pas seulement en l'acquisition d'une somme de connaissances mais qu'elle doit également favoriser une capacité critique quant aux savoirs acquis. En ce sens, au-delà d'une revue des enseignements stricto-sensus, ce texte propose une contribution à la discussion relative à la portée heuristique de l'approche transnationale, initiée depuis quelques années mais pas encore épuisée (Amelina, et al. : 2014 ; Boccagni : 2012 ; Dumitru : 2014 ; Glick Schiller : 2015 ; Green et Waldinger : 2016).

2 L'article revient d'abord brièvement sur les évolutions des paradigmes mobilisés dans l'enseignement de la sociologie des migrations à l'URMIS afin de développer, dans une deuxième partie, une discussion autour de l'avènement de l'approche transnationale, de ses influences et de ses présupposés intellectuels. La troisième partie offre un regard critique sur l'incidence des conditions matérielles de production de la recherche scientifique sur ses orientations paradigmatiques. La place donnée ici à ce débat ne présuppose pas que la thématique transnationale soit dominante dans les formations de l'URMIS; elle n'en est qu'une composante, aux côtés d'autres enseignements (notamment sur les thématiques du racisme, des discriminations, des relations interethniques, etc.). Il ne s'agit donc pas d'une étude exhaustive de l'ensemble des 
contenus des cours dispensés dans les deux formations concernées, mais plutôt d'examiner l'évolution d'une partie de leurs enseignements, afin de comprendre comment un domaine d'étude s'est progressivement restructuré, jusque dans ses apprentissages. C'est donc à partir d'une bonne connaissance de ce matériau ${ }^{3}$ et d'une revue de la littérature que l'auteure choisit de centrer son propos sur les développements récents de l'approche transnationale.

\section{De l'interculturel au transnationalisme}

3 A.Sayad fut un des premier auteurs français à remettre en question l'ethnocentrisme qui a longtemps prévalu dans les études sur les migrations (1977). Tant que l'immigration en France fut dominée par l'arrivée de populations anciennement colonisées, la majorité des travaux des sociologues restaient plus ou moins liés au point de vue de la société dominante sur ces éléments allogènes, récemment sortis de la catégorie "Indigènes ». C'est d'abord leur participation à la sphère économique et, partant, au monde du travail qui a fait l'objet des travaux de recherche portant sur les migrants.

4 Puis la crise pétrolière de 1973, l'arrêt officiel de l'immigration permanente de travail en 1974 et la facilitation du regroupement familial ont conduit à examiner la situation de coprésence sous divers aspects. Les questions liées à l'immigration, l'intégration, la gestion de ces populations par l'État ou encore les relations interculturelles sont devenues prépondérantes dans le débat sociologique. C'est à cette époque que naît à Nice l'Institut d'études et de recherches interethniques et interculturelles (IDERIC), ancêtre de l'URMIS. Ce laboratoire, précurseur en France, formera des étudiants de DEA et de doctorat durant une quinzaine d'années avant de se muer, en 1994, en une nouvelle unité de recherche, associant le CNRS, les universités de Nice Sophia Antipolis et Paris 7 et, plus récemment l'IRD (2008), renouvelant en partie ses approches de la question migratoire et initiant deux masters sur les migrations et les relations interethniques. Ces formations verront leur contenu progressivement évoluer au cours des années quatre-vingt-dix puis deux mille.

5 La chute des régimes communistes, et avec eux celle d'un monde bipolaire, n'est pas étrangère à ce renouveau. En Europe, le processus de diversification des mobilités et l'interdépendance des économies nationales à l'échelle du monde ont débuté très tôt mais l'éclatement du «bloc de l'Est» en 1989 a été décisif et a conduit à orienter l'attention des chercheurs vers les circulations qui se faisaient jour à travers le continent. Le nouveau contexte géopolitique, en même temps qu'il a bouleversé le continent européen, a contraint ses observateurs à en redéfinir les grilles de lectures. Dès lors, la sociologie des migrations s'est muée en deux tendances, toutes deux encore aujourd'hui représentées dans les masters de l'URMIS :

- Un premier courant a pris le parti de redessiner la question de l'intégration pour produire une série de travaux sur le racisme et le maintien des discriminations (De Rudder, Poiret, Vourc'h: 2000), au-delà de la phase de migration (ou d'arrivées numériquement importantes), donnant également un essor nouveau à la sociologie des relations interethniques (Poutignat et Streiff-Fénart : 1995) ou des race relations qui se développaient aux États-Unis.

- Un second a porté son regard sur les mobilités et leur mise en pratique dans des espaces en redéfinition, parfois dits transnationaux, et dans lesquels les déplacements étaient 
grandement facilités par les moyens modernes ${ }^{4}$. À l'URMIS, ce courant a commencé à faire l'objet de cours dès la fin des années quatre-vingt-dix. Dans cette perspective, les migrants devenaient l'illustration humaine de la mondialisation, quitte à en être son coût (Bauman : 1999).

Ce dernier courant, plus longuement discuté ici, a conduit à renouveler les enseignements et a fait émerger une nouvelle génération de chercheurs pour lesquels le lien entre situation postcoloniale et migrations n'avait plus rien d'évident. L'immigré n'était plus forcément maghrébin ou africain ni même issu du tiers-monde et son projet d'installation n'était pas considéré comme allant de soi. En quête de références sur lesquelles appuyer la réflexion naissante, les recherches et les enseignements sur les migrations transeuropéennes ont défini un nouveau cadre conceptuel, appuyé sur des travaux qui n'étaient pas alors « mainstream » en sociologie des migrations et ouvrant davantage la thématique à l'interdisciplinarité.

7 La perspective ouverte par A. Tarrius (1992) et ses collaborateurs (M. Péraldi, E. Témime notamment), dans le prolongement de ceux initiés par des géographes et notamment G. Simon (1995), était une composante nouvelle de l'enseignement de DEA à Nice à partir de la fin des années quatre-vingt-dix. Ces travaux tendaient à s'affranchir du couple immigration/intégration pour proposer un regard davantage centré sur la mobilité et le point de vue des acteurs. Ils apportaient un éclairage sur les «sociétés de migrants ", pointant la complexité de dispositifs dans lesquels se mêlaient activités économiques et relations sociales partagées par des populations aux parcours divers. S’il s'agissait, pour la plupart, de migrants maghrébins, les auteurs s'écartaient définitivement de l'approche classique pour considérer la mobilité comme un objet de recherche en soi.

8 Dans la même veine, Mirjana Morokvasic publie des travaux éclairés ${ }^{5}$ sur les circulations des ressortissants des pays de l'Est, soulignant leur caractère mouvant, pendulaire et la précarité des agencements sociaux et commerciaux dans lesquels ils s'inséraient $(1992,1996)$. On peut penser que la spécialisation antérieure de cette chercheuse n'est pas pour rien dans sa capacité à saisir ces processus émergents. Ses recherches sur les femmes migrantes l'avaient en effet conduit à mettre de côté la figure emblématique du migrant-ouvrier (chef de famille, délégué de sa communauté...) pour se concentrer sur une migration tout aussi importante numériquement mais qui n'apparaissait pas digne d'intérêt pour qui voyait avant tout dans les immigrés des agents dominés du capitalisme industriel. Même si elle a par la suite soulevé les limites de cette notion, on peut considérer que cette auteure a contribué à introduire en France la perspective transnationale.

9 Les chercheurs cités ici ne sont pas les seuls à avoir pris, relativement tôt, de la distance par rapport à l'environnement intellectuel dominant de l'époque ${ }^{6}$, mais ils en sont emblématiques en France et sont devenus des références dans l'enseignement des «nouvelles mobilités ». Ces travaux se caractérisaient notamment par leur divergence avec la perspective fortement déterministe qui marquait de nombreuses recherches de sociologie de l'immigration dans les décennies précédentes. En ce sens, le renouveau de la sociologie des migrations est aussi une conséquence plus ou moins directe de la perte d'influence de l'idéologie marxiste auprès des intellectuels. Sans céder à l'individualisme méthodologique, les travaux sur la circulation migratoire, ont remis le focus sur des sujets dotés d'une certaine marge de manœuvre et, en partie au moins, 
libérés des contraintes structurelles de leur position sociale ou, a minima, tentant de s'en affranchir.

Basés sur l'étude des migrants latinos aux États-Unis, les travaux américains ont constitué une autre ressource des nouveaux enseignements sur les mobilités, aux côtés de recherches sur la mobilité en Afrique (Bredeloup : 1993 ; Marchal et Quesnel : 1997 ; Pliez: 2000). La perception selon laquelle le monde se rétrécissait grâce tant aux moyens de transport, qu'aux autoroutes de l'information et à la multiplication des échanges a conduit la recherche à s'intéresser à des espaces sociaux qui ne se limitaient pas (ou plus) aux frontières politiques et à remettre en question l'hégémonie des États sur la scène internationale (Badie : 1999). Alejandro Portes et d'autres après lui ont fort bien argumenté l'idée selon laquelle les migrants étaient passés en quelques décennies, d'un modèle d'ancrage social fort dans le pays d'immigration au modèle de la communauté transnationale transgressive envers la normativité étatique (GlickSchiller et al.: 1997; Portes: 1996, 2001). Ce qui séduisait - et séduit encore - de nombreux chercheurs dans l'approche transnationale tient à sa capacité à saisir ce qui se joue en divers espaces en même temps, au-delà des territoires bornés par les frontières. Remettant le migrant au centre de la scène, cette perspective permet en outre d'intégrer dans les analyses la capacité d'action et même d'engagement politique d'acteurs qui ne sont pas seulement déterminés par le jeu des contraintes nationales. Par là-même, on a pu aborder les effets de la mondialisation sur la relation des individus aux États. Nina Glick-Schiller, reprenant une problématique de l'École de Chicago, a notamment traité de la question de l'allégeance des migrants transnationaux, non dans un contexte de guerre comme la fondation Carnegie au début du vingtième siècle, mais dans le monde hyper connecté et interdépendant du vingt-etunième siècle (Glick-Schiller et al. : 1997).

11 À partir des années deux mille, l'approche transnationale, venue des États-Unis, est progressivement mobilisée en France où elle offre un cadre conceptuel à la multitude de travaux qui se développent à l'époque autour de la circulation migratoire. Le terme transnational, parfois considéré comme position paradigmatique, d'autre fois seulement comme une réalité dont il faudrait tenir compte, prend désormais une place importante dans les enseignements de sociologie des migrations. Bien qu'il ne s'agisse pas d'une école et que les travaux soient forts divers, cette perspective entend (1) décrire un phénomène nouveau ou en forte augmentation (l'activité transnationale des migrants), (2) proposer une méthodologie qui rompt avec l'ethnocentrisme, (3) redessiner la figure du migrant en lui restituant sa subjectivité et son autonomie.

\section{Rupture épistémologique et figure du migrant contemporain}

12 Cette approche (le transnationalisme) a donné une nouvelle impulsion à la recherche sur les migrations en France. À l'URMIS, à la fin des années quatre-vingt-dix, elle a été introduite dans les formations de DEA de Nice d'une part et de Paris via sa cohabilitation avec Migrinter, d'autre part. Plusieurs doctorats s'y sont inscrits ou l'ont commentée (Fresnoza-Flot : 2008 ; Pian : 2007 ; Potot : 2003) ${ }^{7}$. À cette époque, un certain nombre de chercheurs ont également mis en question la portée novatrice de ce courant (Streiff-Fénart : 2002 ; Waldinger et Fitzgerald : 2004). Dès lors, certains suggéraient que le passage d'un modèle de migrant à un autre était peut-être autant lié à l'évolution des 
migrations elles-mêmes qu'à une attention nouvelle à ces dimensions transnationales de la part des chercheurs (Potot: 2003), postulat progressivement partagé par un nombre grandissant de chercheurs (Boccagni : 2012 ; Lazar : 2011). Bien entendu, les pratiques migratoires ont évolué avec les transformations de leurs environnements, mais la rupture fut plus épistémologique qu'empirique. Tandis que les précurseurs du transnationalisme migrant entendaient définir un nouveau concept pour observer une réalité sociale qu'ils pensaient être nouvelle, celui-ci a eu un impact d'une autre nature : il a conduit à saisir des aspects de la réalité sociale qui, bien qu'existants, restaient jusqu'alors imperceptibles aux yeux des chercheurs. C'est pourquoi, d'une certaine façon, le courant transnational est indissociable, en sociologie des migrations, $\mathrm{du}$ dépassement $\mathrm{du}$ nationalisme méthodologique et participe des mêmes débats (Chavel : 2014; Dumitru : 2014; Wimmer et Glick Schiller : 2003). Toutefois, si la capacité de cette approche à pointer et décrire certaines évolutions dans les pratiques des migrants est attestée, son extension, au-delà du cadre empirique dans lequel elle a été forgée, à l'étude de l'ensemble des migrations que l'on disait nouvelles parce que contemporaines, ne fut pas toujours heureuse.

La figure archétypale du migrant transnational est un commerçant autonome et en constante mobilité, que celle-ci soit physique ou dans ses relations sociales et projections culturelles. Le postulat implicite est qu'il incarne en cela le processus de mondialisation, dans ses dimensions économique, technologique, sociale, etc. La référence presque systématique à cette approche dans les travaux portant sur les migrations contemporaines a eu pour conséquence de laisser croire que le travail des étrangers, indépendant et inscrit dans des dispositifs d'échanges (commerciaux) autorégulés et en grande partie affranchis de l'emprise des États, était la modalité d'insertion économique la plus révélatrice des mutations sociologiques contemporaines. Dit autrement, cela suggère que la mondialisation a eu pour effet de transformer l'immigré ouvrier du secteur secondaire en indépendant ou au moins de considérer qu'elle en a fait un modèle dominant.

Or, s'il a été considéré comme un idéal-type de référence pour toute une génération de chercheurs, il n'y a aucune raison de considérer que le commerçant est devenu majoritaire parmi les migrants ou qu'il aurait vocation à remplacer progressivement le salarié. Les nombreux travaux publiés sur les travailleurs migrants précaires, dans les $3 \mathrm{D}$ jobs $^{8}$ notamment, soulignent l'importance du salariat au sein de cette population (Anderson : 2000; Morice et Potot: 2010). Si les nouvelles mobilités sont toutes concernées par le processus de mondialisation, c'est une erreur de considérer qu'elles s'inscrivent globalement (toujours) dans un processus d'autonomisation des migrants.

Dans ce contexte intellectuel, une dichotomie s'est dessinée à partir des forces agissant dans le champ : d'un côté de nombreux chercheurs s'abreuvaient au transnationalisme et menaient de vastes programmes de recherche sur les transformations des migrations, de l'autre des chercheurs européens, notamment sociologues du travail, s'appliquaient à mettre au jour des processus de domination actuels en s'appuyant sur des référentiels théoriques solides, issus de d'approches néo-marxistes, mais quelque peu délaissés par les jeunes chercheurs.

16 Dans ses formations, à Paris comme à Nice, l'URMIS a eu à cœur de tenir ensemble ces deux dimensions. On considère en effet, dans le prolongement de la réflexion de Saskia Sassen qui distingue des «villes globales " mondialisées et des «villes locales » marginalisées (Sassen : 2002), et inspirés par les travaux d'Andrea Rea (2002), Alain 
Morice (2000), Salvatore Palidda (1999), Maurizio Ambrosini (1999) et bien d'autres encore, qu'il serait fortuit d'aborder la mondialisation au-delà des hiérarchies qu'elle fonde - et partant, des classes sociales- pour traiter des mobilités contemporaines.

Les travaux des pères - et mères - fondateurs du transnationalisme dans sa définition la plus large, cherchant à montrer que les élites n'étaient pas les seules à se mondialiser, ont souligné la capacité de " ceux d'en bas » à faire comme " ceux d'en haut » (Portes : 1999). C'est-à-dire à créer des champs sociaux transnationaux, à établir des ponts entre des aires fortement différenciées et à vivre dans des sphères si connectées que l'on n'y distingue plus l'ailleurs de l'ici (Diminescu : 2005 ; Diminescu, et al. : 2010 ; Nedelcu : 2003). Dans cet univers, les frontières sont transgressées au quotidien et les individus mobilisent de la même façon, qu'ils soient cadres d'entreprise transnationales ou migrants économiques de faible condition, des ressources acquises par l'expérience transnationale.

Une lecture rapide de ces travaux laisse penser que la mondialisation aurait permis d'une certaine façon aux migrants de s'émanciper des structures de domination classiques, celles de l'État comme celles de l'entreprise et que cette capacité s'exprime dans leur rapport aux territoires. Pour parvenir à cette conclusion, il faut pourtant choisir parmi les migrants ceux qui sont susceptibles d'être transposés au modèle des élites. En cela, ce n'est pas un hasard si les migrants décrits par ces précurseurs du courant transnational sont avant tout commerçants: ils sont plus protégés que les autres de la domination des sociétés occidentales. La maladresse de certains chercheurs fut d'étendre la perspective transnationale à l'étude des migrations salariées en ne donnant pas toute sa place aux structures de production dans lesquelles elles s'inséraient et à leurs mutations à l'heure de la globalisation. En effet, si les migrants sont plus mobiles, c'est non seulement parce que le contexte de la globalisation le leur permet, mais c'est également parce que toute insertion économique au bas de l'échelle est aujourd'hui extrêmement précaire (Cahiers de l'URMIS : 2009). Si la mobilité est une ressource (Ma Mung: 1999), pour la majorité elle intervient pour pallier un défaut de stabilité dans l'emploi. Cela permet de revenir sur l'implicite selon lequel l'accentuation de la mobilité témoignerait d'une émancipation pour les migrants.

Cette réflexion n'enlève rien à la pertinence de l'approche transnationale mais invite à ne pas laisser hors du regard sociologique les autres migrants considérant qu'ils ne seraient que les reliquats d'une époque révolue, ou que les commerçants seraient à l'avant-garde des pratiques migratoires contemporaines. Au contraire, les travailleurs étrangers sont aujourd'hui largement insérés dans des processus de production ou de service dans lesquels ils occupent une position salariée ou équivalente ${ }^{9}$. Les conditions d'emploi des étrangers sont au cœur des mutations du monde du travail en Occident et sont susceptibles, en cela, d'éclairer les évolutions de la société (Potot: 2013). C'est pourquoi les formations dispensées à l'URMIS entendent valoriser les apports du transnationalisme tout en conservant une attention particulière aux relations de domination qui caractérisent aujourd'hui comme hier, la position immigrée. Creuser les questions d'autonomie des migrants, leur capacité à saisir des opportunités, percevoir leur mobilité et leurs ancrages multiples comme des formes de résistance, n'interdit pas de reconnaître qu'ils sont simultanément des agents économiques et sociaux pris dans des hiérarchies au sein lesquelles ils occupent les places les plus basses. De ce fait, les questions d'insertion économique, de discrimination et de racisme restent, à côté de la perspective transnationale, au cœur des savoirs transmis à l'URMIS. 


\section{Paradigme dominant et conditions de production de la connaissance}

20 Pour finir, on propose d'examiner les conditions matérielles de production de la connaissance qui ont conforté le paradigme transnational. En effet, à partir des années quatre-vingt-dix, le milieu de la recherche a subi des transformations profondes qui l'ont amené à faire évoluer ses pratiques et, partant, le contenu de ses enseignements. On postule ici que ce sont autant les structures d'opportunités rencontrées par les chercheurs que les débats théoriques qui ont fait du transnationalisme le concept dominant de la recherche sur les migrations jusqu'à ces dernières années.

D'une part, l'évolution des technologies a contribué à repousser les frontières de la recherche. Le développement des transports et la baisse de leur coût ont rendu plus accessibles pour les chercheurs, même étudiants de master, les régions d'origine des migrants et les étapes de leurs parcours. D'un point de vue méthodologique, prendre en compte la bipolarité - ou la multipolarité - des migrations est devenu une façon « normale » de faire du terrain sur les migrations. Il ne s'agit pas d'une révolution : la recherche multisituée est bien antérieure à cette période, mais elle s'est fortement démocratisée dans les années deux mille. Ces facilitations matérielles ont accompagné l'émergence de l'analyse du système-monde (Appadurai : 1996; Marcus: 1995; Wallerstein : 2000) qu'elles ont encouragée en retour à travers l'étude des mobilités. Parallèlement, les outils informatiques ont intensifié la communication entre chercheurs, ce qui a accéléré la diffusion des idées et notamment le transfert de concepts de part et d'autre de l'Atlantique, permettant un dialogue fécond, quoi que pas toujours bien éclairé par les contextes locaux, entre recherches menées en différents points du monde et abordées à travers des "cultures scientifiques" différentes. Ces nouvelles conditions de diffusion de la recherche, notamment la mise en ligne de nombreux travaux, ont sans aucun doute participé à l'adoption du transnationalisme dans les recherches européennes.

D'autre part, les évolutions internes au milieu de la recherche en Europe occidentale ont eu un impact non négligeable sur ses objets et ses méthodes. Tandis que le monde ouvrier passait des grands collectifs industriels à la sous-traitance à grande échelle, le monde académique passait d'une recherche mue par l'évolution, à leur rythme, de ses propres questionnements à une recherche par projets, financée au coup par coup, sur le mode de la concurrence. La construction européenne s'est accompagnée d'une redistribution des budgets dont une partie est désormais gérée au niveau européen à travers les programmes cadres de recherche et développement (PCRD) et plus récemment le programme Horizon 2020. La recherche de partenaires scientifiques internationaux, la valorisation du dialogue entre disciplines et les outputs / délivrables sont devenus des éléments fondamentaux de tout projet.

À côté du contenu problématique, les chercheurs ont intégré ces critères de sélection qui ouvrent la porte à l'obtention de crédits. Au-delà de la seule nécessité de financer des recherches, la soumission de projets et l'obtention de financements sont en quelque sorte devenus des labels qui mettent en avant certains chercheurs pour leur «excellence». Elle leur permet au passage de pallier les difficultés d'embauche des jeunes chercheurs en leur proposant des post-doctorats ou autres contrats temporaires. Même si cela s'est fait - et continue à se faire - au corps défendant d'une grande partie 
de la profession, tous les chercheurs sont affectés par ces pratiques ${ }^{10}$ et cette façon de faire affecte le contenu des recherches. Si le paradigme transnational, et plus largement l'étude des nouvelles mobilités, ont pris un tel essor au cours des années deux mille c'est aussi du fait de cette politique de la recherche.

En effet, l'approche transnationale satisfait particulièrement bien les attendus de ce modèle. Les projets européens et parfois nationaux (ANR) sont évalués de façon anonyme, par des « experts » internationaux pas toujours spécialistes du champ ; il est important alors de présenter des programmes de recherche lisibles par le plus grand nombre. Le terme de transnationalisme (ou ses déclinaisons : migrant transnational, mobilités transnationales, etc.) s'est ainsi imposé comme un marqueur facilement identifiable, en français mais aussi en anglais, au-delà des écoles ou des courants : il renvoie au processus de mondialisation qui parle à tout le monde même s'il n'est jamais défini. D'une certaine façon, le transnationalisme simplifie et dépolitise la recherche sur les migrations aux yeux d'institutions elles-mêmes plus portées par la «bonne gouvernance » que par les débats de société. En français, il en va de même pour les «nouvelles mobilités» ou la «circulation», nettement moins polémiques que «l'immigration », « l'intégration » ou « les travailleurs sans-papiers ».

Cette approche correspond bien, en outre, à l'attendu de l'internationalisation des recherches : en écho à son objet d'étude, elle convoque facilement des chercheurs de nationalités différentes, travaillant sur des terrains éloignés mais connectés par les migrations. Elle se prête également à l'interdisciplinarité dans la mesure où elle fait le lien entre les notions de territoire (plutôt abordé par la géographie), de lien social (cœur de la sociologie), de mondialisation (dans son volet économique) et convoque même les sciences de l'information et de la communication ${ }^{11}$. Par ailleurs, le transnationalisme, comme les nouvelles mobilités, renvoient d'une certaine façon à l'innovation, terme fort des politiques de recherche: il s'agit de travailler sur des processus apparus avec la mondialisation, dans son sillage, suggérant que l'on va forcément y découvrir des innovations sociales puisqu'il s'agit d'une réalité sociale nouvelle.

Enfin, étant donné l'importance politique qu'a pris la thématique des migrations au cours des dernières décennies, il est relativement facile de prévoir des «délivrables » en dehors de la seule sphère académique, en direction des acteurs politiques (même si dans la réalité ces rencontres ne sont pas si fréquentes) ou de la société civile. On est tout à fait là en correspondance, au moins dans les représentations, avec ce que le politique attend des sciences sociales : éclairer les décideurs sur la réalité sociale sans charrier dans ses travaux de bagage idéologique.

\section{Conclusion}

Ce propos n'a pas vocation à délégitimer l'approche transnationale mais, en la soumettant à une certaine déconstruction, à saisir ce qui la constitue pour en conserver les apports, sans aveuglement. Il éclaire ainsi les choix opérés en termes de formation à URMIS : les différents volets de l'enseignement visent à initier les étudiants à un large corpus de travaux afin de leur offrir les moyens de comprendre et de participer aux débats contemporains de la recherche sur les migrations, au-delà des pré-notions dont ils sont souvent porteurs en tant que citoyens et sans exclusive disciplinaire. 


\section{BIBLIOGRAPHIE}

Ambrosini, Maurizio (1999) Travailler dans l'ombre. Les immigrés dans l'économie informelle, Revue Européenne des Migrations Internationales, vol. 15, pp. 95-121.

Amelina, Anna ; Nergiz, Devrimsel D. ; Faist, Thomas ; Glick Schiller, Nina (2014) Beyond Methodological Nationalism : Research Methodologies for Cross-Border Studies, New York, Routledge, $260 \mathrm{p}$.

Anderson, Bridget (2000) Doing the dirty work? The global politics of domestic labour, London, Zed Books, $224 \mathrm{p}$.

Appadurai, Arjun (1996) Modernity at Large : Cultural Dimensions of Globalization, Minneapolis, University of Minnesota Press, 248 p.

Badie, Bertrand (1999) Le retournement du monde, Paris, Presses de Sciences Po, $252 \mathrm{p}$.

Bauman, Zygmund (1999) Le coût humain de la mondialisation, Paris, Hachette Littératures, 180 p.

Boccagni, Paolo (2012) Revisiting the "Transnational” in Migration Studies : A Sociological Understanding, Revue européenne des migrations internationales, vol. 28, $\mathrm{n}^{\circ} 1$, pp. 33-50.

Bredeloup, Sylvie (1993) Les migrants du fleuve Sénégal : À quand la « Diams' pora » ?, Revue européenne des migrations internationales, vol. 9, n 3, pp. 205-232.

Cahiers de l'URMIS (2009) Circulation migratoire et insertions économiques précaires en Europe, $\mathrm{n}^{\circ} 9$, juin 2009, https://journals.openedition.org/urmis/763.

Chavel, Solange (2014) De la migration à la mobilité : comment aller au-delà du nationalisme méthodologique ?, Raisons politiques, vol. 54, $\mathrm{n}^{\circ} 2$, pp. 53-66.

De Rudder, Véronique ; Poiret, Christian ; Vourc'h, François (2000) L'Inégalité raciste. L'universalité républicaine à l'épreuve, Paris, Presses Universitaires de France, $224 \mathrm{p}$.

Diminescu, Dana (2005) Le migrant connecté : pour un manifeste épistémologique, Migrations société, vol. 17, n 102, pp. 275-292.

Diminescu, Dana ; Renault, Matthieu ; Jacomy, Mathieu ; D'Iribarne, Christophe (2010) Le web matrimonial des migrants, Réseaux, vol. 159, n 1, pp. 15-56.

Dumitru, Speranta (2014) Qu'est-ce que le nationalisme méthodologique ?, Raisons politiques, vol. $54, \mathrm{n}^{\circ} 2$, p. $9-22$.

Fresnoza-Flot, Asuncion (2008) Migration, genre et famille transnationale :l'exemple des mères migrantes philippines en France, Paris, Université Paris 7, 499 p.

Th. Doct. : sociologie : Paris : 2008.

Glick Schiller, Nina (2015) Explanatory frameworks in transnational migration studies : the missing multi-scalar global perspective, Ethnic and Racial Studies, vol. 38, nº 13, pp. 2275-2282.

Glick-Schiller, Nina ; et al. (1997) From immigrant to transmigrant : Theorizing transnational migration, Sozial Welt. Sonderband, vol. 12, pp. 121-140.

Green, Nancy ; Waldinger, Roger (2016) A Century of Transnationalism. Immigrants and Their Homeland Connections, Urbana, University of Illinois Press, 292 p.

Lazar, Andreea (2011) Transnational migration studies. Reframing sociological imagination and research, Journal of comparative research in Anthropology and Sociology, $\mathrm{n}^{\circ}$ 2, pp. 69-83. 
Ma Mung, Emmanuel (1999) La dispersion comme ressource, Cultures et conflits, $\mathrm{n}^{\circ} 33-34$, pp. 89-103.

Ma Mung, Emmanuel ; Dorai, Kamel ; Boyer, Françoise ; Hily, Marie-Antoinette (1998) La circulation migratoire. Bilan des travaux, Migrations Études, $150 \mathrm{p}$.

Marchal, Jean-Yves ; Quesnel, André (1997) Dans les vallées du Burkina Faso, l'installation de la mobilité, in Gastellu, J.-M. ; Marchal, J.-Y. (dir.) La ruralité dans les pays du Sud à la fin du XXe siècle, Paris, ORSTOM, pp. 595-614.

Marcus, George E. (1995) Ethnography in/of the World System : the Emergence of Multi-Sited Ethnography, Annual Review of Anthropology, vol. 24, pp. 95-117.

Morice, Alain (2000) Recherches sur le paternalisme et le clientélisme contemporains : méthodes et interprétations, Paris, École des Hautes Études en Sciences Sociales (EHESS), 228 p.

HDR : Géogr. : Paris : 2000.

Morice, Alain ; Potot, Swanie (dir.) (2010) De l'ouvrier immigré au travailleur sans papier. Les migrants dans la modernisation du salariat, Paris, Karthala, 336 p.

Morokvasic, Mirjana (1996) Entre l'Est et l'Ouest, des migrations pendulaires, in Migrants. Les nouvelles mobilités en Europe, Paris, L'Harmattan, pp. 119-157.

Morokvasic, Mirjana (1992) Une migration pendulaire : les polonais en Allemagne, Hommes et migrations, $\mathrm{n}^{\circ}$ 1155, pp. 31-36.

Nedelcu, Mihaila (2003) E-communautarisme ou l'impact de l'internet sur le quotidien des migrants, in Diminescu, D. (dir.) Visibles mais peu nombreux. Les circulations migratoires roumaines, Paris, Éditions de la Maison des Sciences de l'Homme, pp. 325-340.

Palidda, Salvatore (1999) La criminalisation des migrants, Actes de la recherche en sciences sociales, vol. $129, \mathrm{n}^{\circ} 1$, pp. 39-49.

Pian, Anaïk (2007) Les Sénégalais en transit au Maroc. La formation d'un espace-temps de l'entre-deux aux marges de l'Europe, Paris, Université Paris 7, $486 \mathrm{p}$.

Th.Doct. : Sociologie. : Paris : 2007.

Pliez, Olivier (2000) Le Sahara libyen dans les nouvelles configurations migratoires, Revue européenne des migrations internationales, vol. 16, $\mathrm{n}^{\circ}$ 3, pp. 165-181.

Portes, Alejandro (2001) Introduction : the debates and significance of immigrant transnationalism, Global Networks, vol. 1, n 3, [consulté le 27/07/2018] [Disponible sur Internet].

Portes, Alejandro (1999) La mondialisation par le bas. L'émergence des communautés transnationales, Actes de la recherche en sciences sociales, vol. 129, $\mathrm{n}^{\circ}$ 1, pp. 15-25.

Portes, Alejandro (1996) Global Villagers : The Rise of Transnational Communities, The American Prospect, vol. 7.

Potot, Swanie (2013) Construction européenne et migrations de travail : le renouvellement des modes de mobilisation de la main-d'œuvre étrangère, Revue Européenne des Sciences Sociales, vol. 51, $\mathrm{n}^{\circ} 1$, pp. 7-32.

Potot, Swanie (2003) Circulation et réseaux de migrants roumains: Une contribution à l'étude des nouvelles mobilités en Europe, Nice, Université Nice Sophia Antipolis, 387 p.

Th.Doct. : Sociologie : Nice : 2003.

Poutignat, Philippe ; Streiff-Fénart, Jocelyne (1995) Théories de l'ethnicité. Suivi de Les groupes ethniques et leurs frontières (F.Barth), Paris, Presses Universitaires de France, 270 p. 
Réa, Andrea (2002) Le travail des sans-papiers et la citoyenneté domestique, in Péraldi, M. (dir.)

La fin des norias? Réseaux migrants dans les économies marchandes en Méditerranée, Paris,

Maisonneuve et Larose ; MMSH, pp. 450-478.

Sassen, Saskia (2002) Global Networks, Linked Cities, London, Routledge, 376 p.

Sayad, Abdelmalek (1977) Les trois âges de l'émigration algérienne en France, Actes de la recherche en sciences sociales, vol. $15, \mathrm{n}^{\circ} 1$, pp. 59-79.

Streiff-Fénart, Jocelyne (2002) Transnationalité et ethnicité, in Péraldi, M. (dir.) La fin des norias ? Réseaux migrants dans les économies marchandes en Méditerranée, Paris, Maisonneuve et Larose, pp. 489-495.

Simon, Gildas (1995) Géodynamique des migrations internationales dans le monde, Paris, PUF, 429 p.

Tarrius, Alain (1992) Les fourmis d'Europe, Paris, L'Harmattan, 207 p.

Waldinger, Roger ; Fitzgerald, David (2004) Transnationalism in Question, Amercian Journal of Sociology, vol. 109, pp. 1177-1195.

Wallerstein, Immanuel (2000) Globalization or the Age of Transition. A Long-Term View of the Trajectory of the World-System, International Sociology, vol. 15, pp. 249-265.

Wihtol de Wenden, Catherine ; De Tinguy, Anne (1995) L'Europe et toutes ses migrations, Bruxelles, Éditions complexe, $173 \mathrm{p}$.

Wimmer, Andreas ; Glick Schiller, Nina (2003) Methodological Nationalism, the Social Sciences, and the Study of Migration: An Essay in Historical Epistemology, International Migration Review, vol. 37, $\mathrm{n}^{\circ}$ 3, pp. 576-610.

\section{NOTES}

1. Colloque international Penser les migrations pour repenser la société, 21-24 Juin 2016, Université de Poitiers, Migrinter

2. L'URMIS a été créée en 1994.

3. L'auteure enseigne dans le master de l'URMIS Nice depuis 2007 et était responsable du M2R de 2012 à 2017.

4. Voir illustration : évolution du recours au terme « Transnational » dans les publications.

5. Au début des années quatre-vingt-dix, les publications sur les migrations venues de l'Est ont été légion mais peu étaient étayées par une connaissance de la recherche sur les migrations et des terrains d'enquête sérieux.

6. Voir notamment les travaux de C. Wihtol de Wenden et A. De Tinguy (1995) ou la synthèse proposée par des chercheurs de Migrinter en 1998 (Ma Mung, et al. : 1998).

7. Merci à Asuncion Fresnoza-Flot, Fanny Jedlicki et Anaïk Pian pour leur contribution à la réécriture des premières lignes de ce paragraphe en mars 2019, afin de corriger certaines inexactitudes.

8. Dirty, Dangerous and Demeaning.

9. Les nouveaux statuts de travailleur indépendant ou liés à la sous-traitance remplacent une partie du salariat mais laisse le travailleur dans une relation de subordination face à son donneur d'ordre à peu près équivalente à celle du salarié.

10. En 2015, 7.000 pré-projets ont été déposés pour le seul appel d'offre blanc de l'ANR avec un taux de succès de $8 \%$ pour les SHS.

11. Voir par exemple le programme commun MSH-Telecom ParisTech sur Le migrant connecté dirigé par Dana Diminescu. 
INDEX

Index géographique : France

Mots-clés : enseignement supérieur, sociologie

\section{AUTEUR}

\section{SWANIE POTOT}

sociologue, chargée de recherche CNRS, Unité de recherche Migrations et société URMIS (UMR 8245), responsable du M2R Migrations et altérités à l'Université de Nice

swanie.potot@cnrs.fr 